\title{
Structural damage detection using MAC-Fast Multi Swarm Optimization technique (MAC- FMSO)
}

\author{
Richard Frans ${ }^{1}$, and Yoyong Arfiadi ${ }^{2, *}$ \\ ${ }^{1}$ Department of Civil Engineering, Universitas Atma Jaya Makassar, Indonesia \\ ${ }^{2}$ Department of Civil Engineering, Universitas Atma Jaya Yogyakarta, Indonesia
}

\begin{abstract}
Damage detection of structures is important to ensure the service of civil engineering infrastructures. Many techniques have been developed for damage detection of structures in the literature. In this paper a new technique, a so called MAC-FMSO technique, is developed by using a combination of Modal Assurance Criterion (MAC) and Fast Multi Swarm Optimization (FMSO) techniques. By using this method, not only the damage location can be assured, but also the loss of strength and loss of stiffness of structures might be quantified. The first stage is to obtain the damaged member's location by using second level of damage detection techniques, such as mode shape curvature method, damage locating vector, or strain energy method. After obtaining the damaged member, the MACFMSO technique is used to obtain the loss of stiffness from the candidate of damaged members. The results show that the MAC-FMSO algorithm has the ability to predict the loss of stiffness in the damaged members. In this case, the MAC-FMSO technique can be considered as an efficient and effective tool at the third level of damage detections. This is because this technique successfully measures the loss of strength or loss of stiffness of the damage member or part of structures. At the end of the paper, the MAC-FMSO technique is applied to predict the location of damage and loss of stiffness of three different structures. The first application is a simply supported beam with three different damage scenarios; the second application is a 13-bar plane truss structure with two different scenarios, and the third application is a shear building structure with three different scenarios. The results of simulation show that the MAC-FMSO technique can accurately predict the loss of stiffness of the damaged member as well as the location of the damage.
\end{abstract}

Keywords: damage identification, loss of stiffness, fast multi swarm optimization, modal assurance criterion, MAC-FMSO

\footnotetext{
* Corresponding author: yoyong@mail.uajy.ac.id
} 


\section{Introduction}

Damage detection of structures can be classified into several levels of identification: (a) level 1: detecting the presence of damage, (b) level 2: locating the damaged members, (c) level 3: determining the loss of strength or stiffness reduction of the damaged members, and (d) level 4: predicting the remaining life time. This paper discusses a simple technique on how to obtain the loss of stiffness of damaged members by using the so called MAC-FMSO algorithm. The MAC-FMSO algorithm can be categorized as a third level of damage detection method. This is because the ability of MAC-FMSO to find the damaged member or damaged part of the structure, and determine the stiffness reduction of the damaged members. The MAC-FMSO consists of two stages in order to obtain the loss of stiffness of the damaged member. The first stage is to use second level of damage detection method to obtain the location of the damage member (e.g.: mode-shape curvature method, damage locating vector method, strain energy method, etc.). The second stage is to use modal assurance criterion (MAC) combined with fast multi swarm optimization (FMSO) to get the stiffness reduction of the damaged members.

\section{Modal Assurance Criterion (MAC)}

One of the techniques that is used to measure the similarity of two mode shapes is modal assurance criterion (MAC). The modal assurance criterion was originated due to the need for quality assurance indicator of the modal vectors obtained from the experimental testing that are estimated from measured frequency response function [1]. The MAC is to provide a statistical measure of consistency between estimates of a modal vector. The resulting MAC is between 0 and 1 . If the modal assurance criterion has a value near zero, this means that the modal vectors are not consistent. On the other hand if the modal assurance criterion has a value near unity, this is an indication that the modal vector are consistent [1]. Because of MAC is based on the minimization of the squared error between two vector spaces, therefore the MAC is sensitive to large differences between comparative values and insensitive to small comparative values.

In terms of mode shape of damaged structure $\left(\phi_{d}\right)$ and mode shape of predicted structural model $\left(\phi_{a}\right)$, the MAC can be defined as:

$$
\left.M A C_{j}=\frac{\left|\left\{\phi_{a}\right\}_{j}^{T}\left\{\phi_{d}\right\}_{j}\right|^{2}}{\left(\left\{\phi_{a}\right\}_{j}^{T}\left\{\phi_{a}\right\}_{j}\right)\left(\left\{\phi_{d}\right\}_{j}^{T}\left\{\phi_{d}\right\}_{j}\right.}\right)
$$

where $\left\{\phi_{a}\right\}_{j}$ is mode shape vector of predicted structure for $j$-mode, and $\left\{\phi_{d}\right\}_{j}$ is mode shape vector of damaged structure for $j$-mode. If the mode shape is identical or similar, the MAC value is 1 , otherwise if the mode shape has no correlation, the MAC value is zero.

\section{Fast Multi Swarm Optimization (FMSO)}

Fast multi swarm optimization (FMSO) that is used in this paper follows the development in [2], from the particle swarm optimization (PSO) technique which was first proposed by [3]. The difference between PSO and FMSO is that the update position and velocity not only depend on equation (2) and (3) but is also influenced by equation (5) as follows:

$$
v_{i, j}(t+1)=w v_{i, j}(t)+c_{1} R_{1}\left(p_{b e s t, i, j}-x_{i, j}(t)\right)+c_{2} R_{2}\left(g_{b e s t, i, j}-x_{i, j}(t)\right)
$$




$$
x_{i, j}(t+1)=x_{i, j}(t)+v_{i, j}(t+1)
$$

where: $v_{i, j}(t+1)$ is the updated velocity of particle, $x_{i, j}(t+1)$ is the updated location of particle, $t$ represents the iteration- $t, p_{\text {best }, i, j}$ is local best location of particle at $t$-iteration, and $g_{b e s t, i, j}$ is global best location of particle at $t$-iteration. $R_{1}$ and $R_{2}$ are random numbers from interval $0-1, c_{1}$ and $c_{2}$ are particle acceleration constants. In this paper, $c_{1}=$ $c_{2}=2$, and $w$ is positive inertia weight coefficient as a function of $\rho_{\min }$ and $\rho_{\max }$ as follows:

$$
\begin{gathered}
w_{(t)}=\rho_{\max }-\left(\left(\rho_{\max }-\rho_{\min }\right) t / \text { max } t\right) \\
x_{i, j(t+1)}=x_{i, j(t)}+\left(v_{i, j(t)} \exp (\delta)\right) \delta_{i}
\end{gathered}
$$

where $\delta$ and $\delta_{i}$ denote Cauchy random numbers.

By adding the update equation (5), PSO greatly increases the probability of escaping from the local optimum because the variance of Cauchy distribution is infinite. In this case, Cauchy mutation makes the particles to have a long jump.

In addition, FMSO also uses crossover operation, in order to enhance the capability for searching the optimum solution. For crossover operation, if rand() $<q_{c}$, the crossover operation is taken as follows:

$$
\begin{aligned}
& x_{i, j(t+1)}=(1-\alpha) x_{i, j(t)}+\alpha p_{\text {best }, i, j} \\
& v_{i, j(t+1)}=\operatorname{rand}()\left(p_{\text {best }, i, j}-x_{i, j(t)}\right)
\end{aligned}
$$

where: $\alpha$ is random number with $0-1$ interval, and $q_{c}$ is crossover rate.

\section{Fitness and penalty functions}

The fitness function is the summations of MAC value from $n$-modes considered of two different mode shapes, i.e., $\phi_{a}$ and $\phi_{d}$. The value of MAC interval is between 0 (no correlations) and 1 (identical). Because in the optimization, we seek the minimum fitness, while the purpose of MAC is to maximize the strong correlation, hence to maximize the function, the fitness should be modified as follow:

$$
F=\frac{1}{\sum_{j=1}^{n} M A C_{j}}
$$

where $F$ is the fitness value, MAC is modal assurance criterion of two mode shapes compared, and $n$ is the number of mode shapes to be considered.

In this paper, the first five mode-shapes were considered, hence the fitness value in equation (8) has interval range from 0.2 to $\infty$. If the fitness equals to 0.2 , it represents the two modes are identical; while if the fitness is $\infty$, this is an indication that no correlation between the two mode-shapes.

The penalty function is activated while one of the particle is less than 0 or larger than 1 . This is because particle represents the loss of stiffness, which is in the interval between 0 and 1. Consequently, when this is the case, the fitness value automatically becomes a maximum value that can be accepted by the program. Fig. 1 shows the step of MAC-FMSO algorithm. 


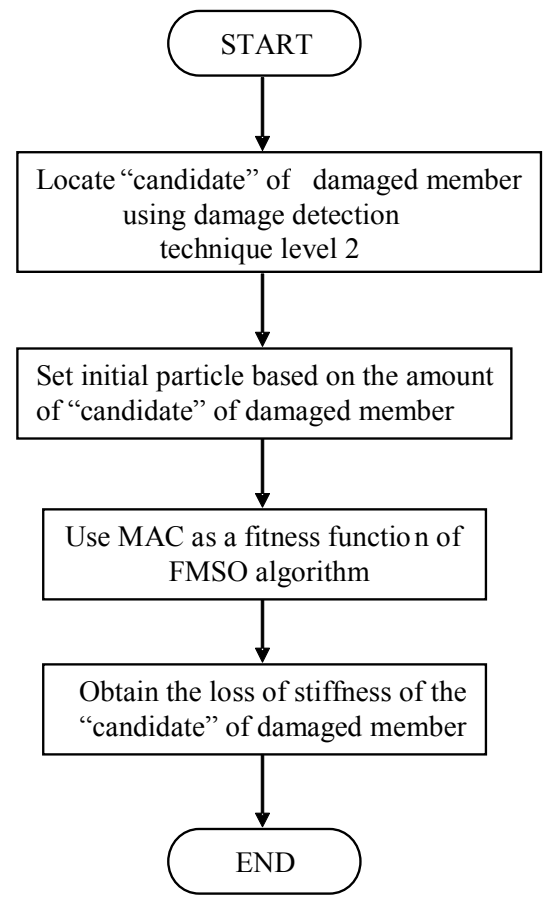

Fig. 1. Procedure of MAC-FMSO for damage detection

\section{Case studies}

\subsection{Simply supported beam model}

The first case study is a simple beam as shown in Figure 2. This model is taken from [4] with some modifications in the damage scenarios. The damage scenarios can be seen in Table 1, while the structural properties of the beam are shown in Table 2. In this example, the loss of stiffness is represented by reducing the modulus of elasticity of the damaged member. The structure is divided into 20 parts. The stiffness matrix method is used for structural analysis.

Table 1. Damage scenarios of beam structure

\begin{tabular}{|c|c|c|c|c|c|}
\hline \multicolumn{2}{|c|}{$\begin{array}{c}\text { First Scenario (Single } \\
\text { Damage) }\end{array}$} & \multicolumn{2}{c|}{$\begin{array}{c}\text { Second Scenario (Double } \\
\text { Damage) }\end{array}$} & \multicolumn{2}{c|}{$\begin{array}{c}\text { Third Scenario (Triple } \\
\text { Damage) }\end{array}$} \\
\hline $\begin{array}{c}\text { Damaged } \\
\text { Member }\end{array}$ & $\begin{array}{c}\text { Loss of } \\
\text { Stiffness }\end{array}$ & $\begin{array}{c}\text { Damaged } \\
\text { Member }\end{array}$ & $\begin{array}{c}\text { Loss of } \\
\text { Stiffness }\end{array}$ & $\begin{array}{c}\text { Damaged } \\
\text { Member }\end{array}$ & $\begin{array}{c}\text { Loss of } \\
\text { Stiffness }\end{array}$ \\
\hline \multirow{2}{*}{10} & & 10 & $50 \%$ & 4 & $70 \%$ \\
\cline { 3 - 6 } & $50 \%$ & 15 & $75 \%$ & 10 & $50 \%$ \\
\cline { 3 - 6 } & & & & 15 & $75 \%$ \\
\hline
\end{tabular}

Following Fig.1, the first step is to locate the damaged member, by using level-2 of damage detection technique, to eliminate the healthy member from the "candidate" of the damaged members as the particle in the swarm. 
Modal curvature mode shape method [5] was used to locate the damaged member. The mode shape index (MSI) of the first damage scenario is shown in Fig. 3. For this case, the first five natural frequencies and mode shapes were taken to locate the "candidate" of the damaged member (part) using mode shape curvature method (MSC).

Table 2. Section and material properties of beam

\begin{tabular}{|l|l|}
\hline \multicolumn{1}{|c|}{ Properties } & Initial Condition \\
\hline Width $(\mathrm{mm})$ & 20 \\
\hline Height $(\mathrm{mm})$ & 200 \\
\hline Area section $\left(\mathrm{mm}^{2}\right)$ & 4000 \\
\hline Modulus of elasticity $(\mathrm{MPa})$ & 200000 \\
\hline
\end{tabular}

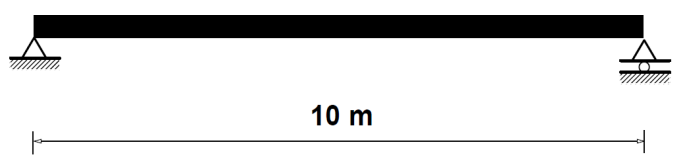

Fig. 2. Simply supported beam

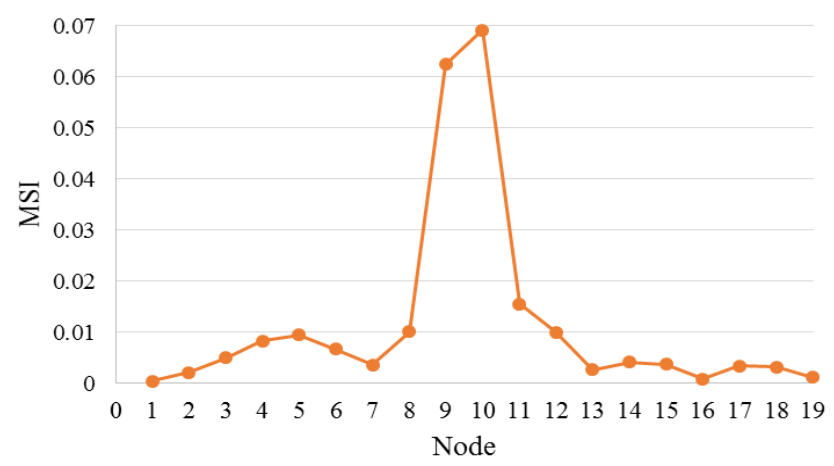

Fig. 3. MSI for first damage scenario

Table 3. Result of stiffness reduction using MAC-FMSO for simply supported beam

\begin{tabular}{|c|c|c|c|}
\hline \multicolumn{4}{|l|}{ First scenario } \\
\hline "Candidate" of damaged member/part & \multicolumn{3}{|c|}{10} \\
\hline Stiffness reduction result using MAC-FMSO & \multicolumn{3}{|c|}{$50 \%$} \\
\hline \multicolumn{4}{|l|}{ Second scenario } \\
\hline "Candidate" of damaged member/part & \multicolumn{2}{|l|}{10} & 15 \\
\hline Stiffness reduction result using MAC-FMSO & \multicolumn{2}{|c|}{$50 \%$} & $75 \%$ \\
\hline \multicolumn{4}{|l|}{ Third scenario } \\
\hline "Candidate" of damaged member/part & 4 & 10 & 15 \\
\hline Stiffness reduction result using MAC-FMSO & $70 \%$ & $50 \%$ & $75 \%$ \\
\hline
\end{tabular}


After locating the "candidate" of the damaged member, the MAC-FMSO was used to find the loss of stiffness of the "candidate" of damaged members. Table 3 shows the result of MAC-FMSO for each damage scenario.

In order to ensure the convergence, the program was run for four times for each damage scenario, with different lower and upper bounds as shown in Table 4. The parameters of optimization using FMSO are: maximum iteration $=100$, number of particles $=10$, maximum velocity $\quad=100$, crossover rate $=0.8, \rho_{\max }=0.9$, and $\rho_{\min }=$ 0.4 .

Table 4. Lower bound and upper bound limit

\begin{tabular}{|c|c|c|}
\hline Run & Lower Bound & Upper Bound \\
\hline 1 & 0 & 1 \\
\hline 2 & 0 & 100 \\
\hline 3 & -10 & 0 \\
\hline 4 & -10 & 100 \\
\hline
\end{tabular}

Fig. 4 shows the fitness value of each iteration for first, second, and third scenario, respectively. From Fig. 4 it is clear that MAC-FMSO can accurately predict loss of stiffness of the damaged member/part for all damage scenarios.

\subsection{Plane truss model}

The second structure considered for MAC-FMSO application is a 13-bar plane truss structure as can be seen in Fig.5. This structure is taken from [4]. Two damage scenarios were considered as follows: (a) a single damage member with loss of stiffness in member11 is $75 \%$ from initial stiffness, and (b) a multiple damage with losses of stiffness in member- 8 and member- 11 are $50 \%$ and $75 \%$, respectively. The stiffness matrix method is used to develop the model.

Fig. 6 shows the normalized stress index of first and second damage scenarios by using damage locating vector proposed by [6]. According to [6] member that has normalized stress index value is almost to zero can be considered as a damaged member. From Fig. 6(a) member-11 can be considered as a damaged member, while From Fig. 6(b) and member 8,11 , and 12 might be categorized as a damaged member. 


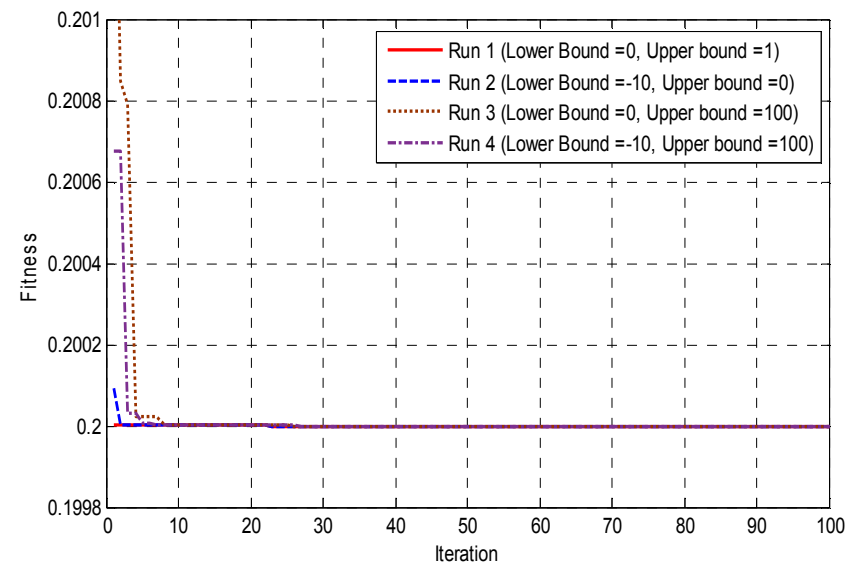

(a)

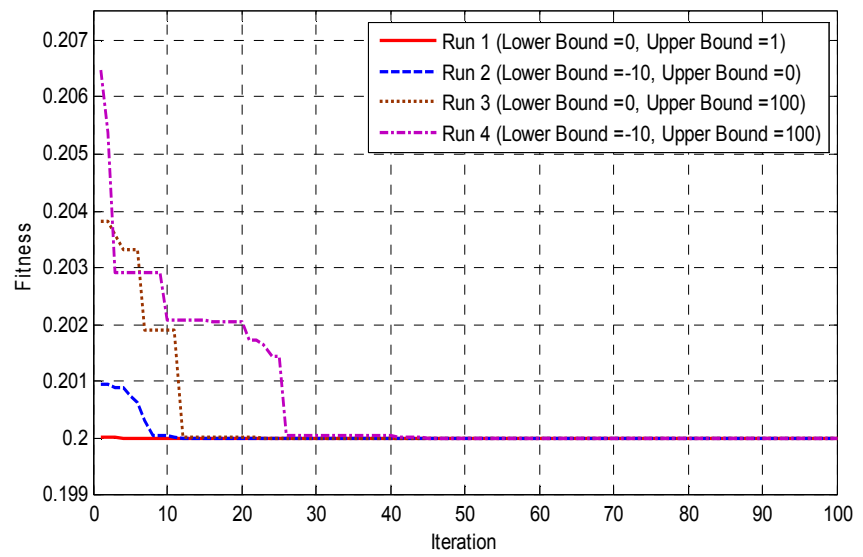

(b)

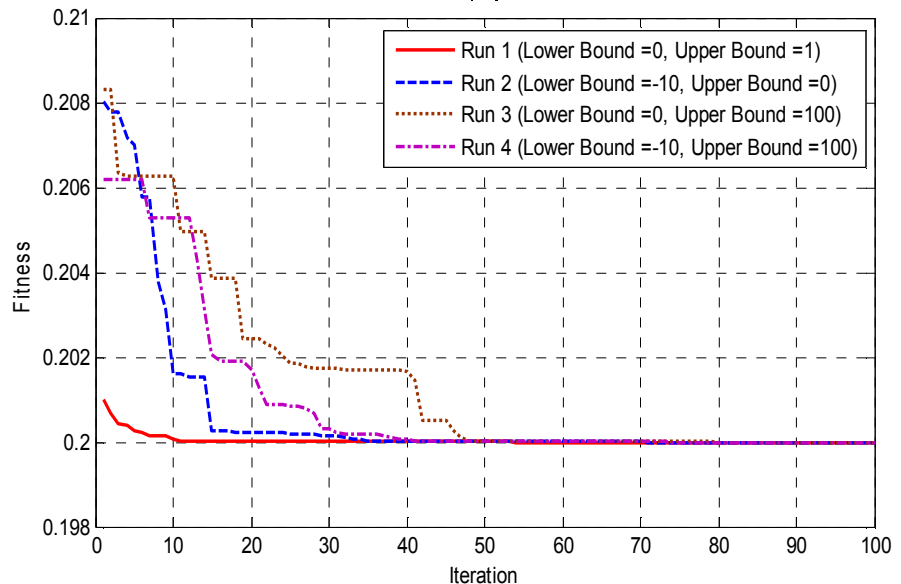

(c)

Fig. 4. Fitness degradation for (a) first scenario, (b) second scenario, and (c) third scenario for simply supported beam 


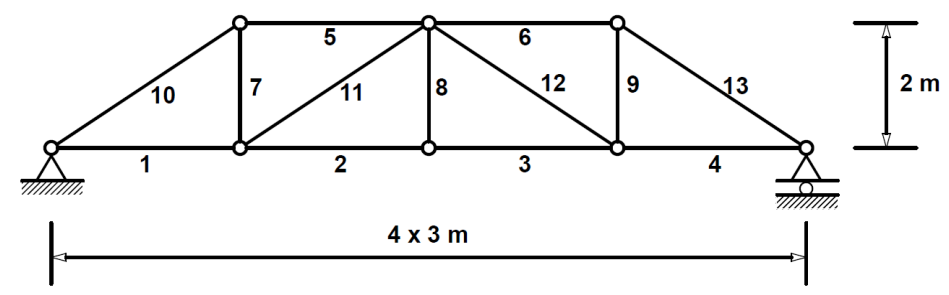

Fig. 5. 13-bar plane truss

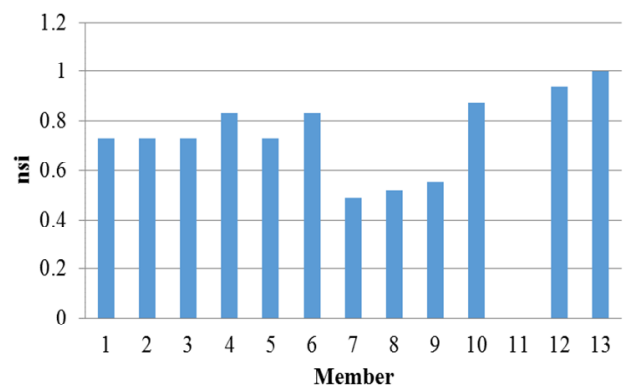

(a)

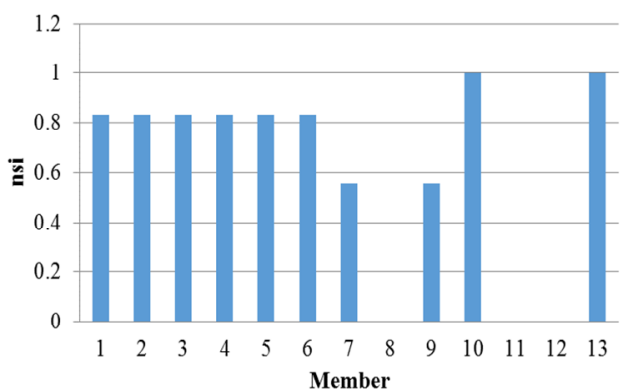

(b)

Fig. 6. Normalized stress index (nsi) for (a) first damage scenario, (b) second damage scenario

After having "candidate" of the damaged members, MAC-FMSO was applied to obtain the loss of stiffness of the damaged member candidate. Lower and upper bound candidates, as well as parameters of FMSO that are used for this case are similar to the first case (simply supported beam). In addition, the program was also run four times to ensure the convergence of the result.

Table 5 shows the results of MAC-FMSO. It can be shown that the MAC-FMSO can accurately predict the stiffness degradation for all damage scenarios. The results also shows another advantage of the MAC-FMSO to identify the multiple damage members. As can be seen in Fig. 6(b), member 12 is also identified as a damaged member by using damage locating vector method. However, the results of MAC-FMSO in Table 5 accurately predict that there is no stiffness reduction in member 12 .

Table 5. Result of stiffness degradation using MAC-FMSO for plane truss structure

\begin{tabular}{|c|c|c|c|}
\hline \multicolumn{4}{|l|}{ First scenario } \\
\hline "Candidate" of damaged member/part & \multicolumn{3}{|c|}{11} \\
\hline Stiffness reduction result using MAC-FMSO & \multicolumn{3}{|c|}{$75 \%$} \\
\hline \multicolumn{4}{|l|}{ Second scenario } \\
\hline "Candidate" of damaged member/part & 8 & 11 & 12 \\
\hline Stiffness reduction result using MAC-FMSO & $50 \%$ & $75 \%$ & $0 \%$ \\
\hline
\end{tabular}




\subsection{Shear building model}

The third example to show the effectiveness of the MAC-FMSO is a shear building model discussed in [6]. Mass and stiffness of each story can be seen in Fig.7. Three different scenarios were considered as follows:
a. a single damage with the loss of stiffness of the sixth story is $60 \%$ from initial stiffness,
b. a double damage with the losses of stiffness in the second and sixth story are $50 \%$ and $40 \%$, respectively,
c. a triple damage with the losses of stiffness in the second, sixth, and ninth story are $70 \%, 55 \%$, and $40 \%$, respectively.

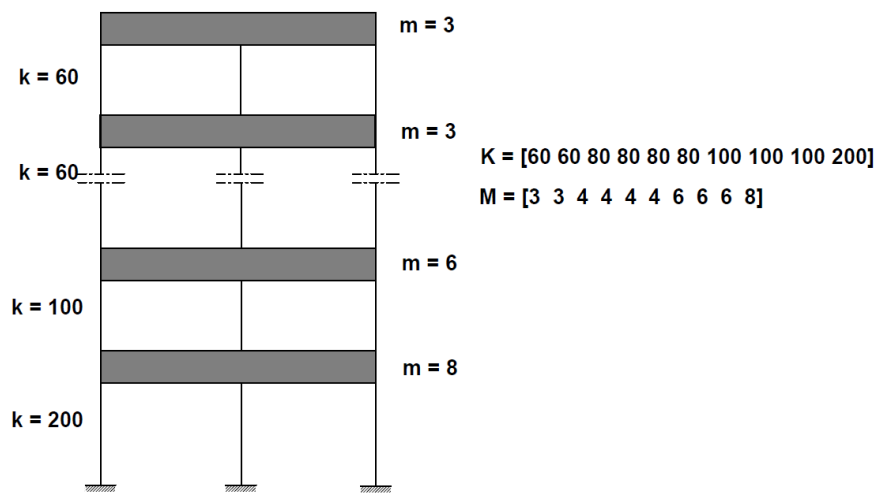

Fig. 7. Shear building properties

The mode shape curvature method was used for this case, and three modes were considered in this simulation. Fig. 8 shows the MSI of the second damage scenario. By observing the MSI, it is clear from Fig. 8 that the damage was occurred in the second and sixth stories.

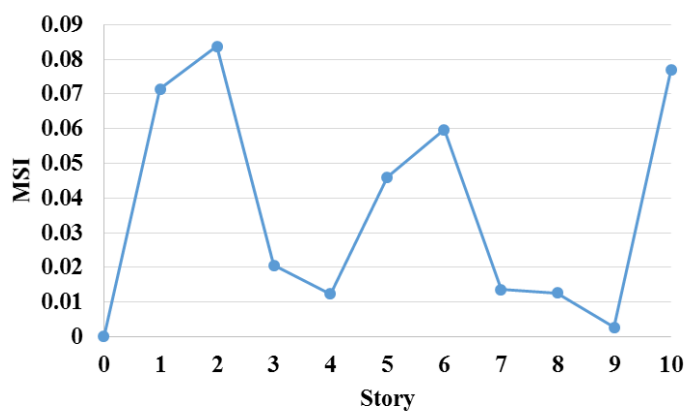

Fig. 8. Mode shape index of shear building for each story

The MAC-FMSO similar to the previous example was used to obtain the loss of stiffness of the structure. The result of simulation is presented in Table 6. From Table 6 it is clear that the MAC-FMSO can accurately predict the loss of stiffness of the structure.

\section{Conclusions}

The MAC-FMSO method is discussed for identification of structural damage. By using MAC-FMSO, the location of the damage as well as the loss of stiffness can be identified. 
The MAC-FMSO consists of two stages. The first stage is finding the damaged member's location by using any of the second level of damage detection techniques, such as mode shape curvature method, damage locating vector, and strain energy method. After obtaining the location of the damaged member, the MAC-FMSO then is used to obtain the loss of stiffness from the "candidate" of the damaged members. The results show that MACFMSO has accurately predict the loss of stiffness of the damaged member/part of structures. Therefore, the MAC-FMSO can be considered as a third level of damage detection method, because it can accurately predict the loss of stiffness of the damaged members.

Table 6. Result of story stiffness degradation using MAC-FMSO for shear building case

\begin{tabular}{|l|c|c|c|}
\hline \multicolumn{3}{|c|}{ First scenario } & \multicolumn{3}{|c|}{6} \\
\hline "Candidate" of damaged story & 2 & 6 \\
\hline Stiffness reduction result using MAC-FMSO & \multicolumn{3}{c|}{} \\
\hline \multicolumn{3}{|c|}{ Second scenario } \\
\hline "Candidate" of damaged story & $50 \%$ & $40 \%$ \\
\hline Stiffness reduction result using MAC-FMSO & 50 . & \\
\hline \multicolumn{3}{|c|}{} \\
\hline "Candidate" of damaged story & 2 & 6 & 9 \\
\hline Stiffness reduction result using MAC-FMSO & $70 \%$ & $55 \%$ & $40 \%$ \\
\hline
\end{tabular}

\section{References}

1. R.J. Allemang, DL. Brown, Proc. of the $1^{\text {st }}$ Int. Modal Analysis Conf., 110-116 (1982)

2. Q. Zhang, C. Li, Y. Liu, L. Kang, Proc. of Advances in Computation and Intelligence, Springer-Verlag, Berlin Heidelberg, 344-352 (2007)

3. J. Kennedy, R.C. Eberhart. Proc. of the 1995 IEEE Int. Conf. on Neural Networks. IEEE Service Center, Piscataway, 1942-1948 (1995)

4. R. Frans, Y. Arfiadi, H. Parung, Procedia Engrg., 1263-1271 (2017)

5. A. K. Pandey, M. Biswas, M. M. Samman, J. of Sound and Vibr., 145, 312-332 (1991)

6. D. Bernal, COST F3, Madrid, Spain, 223-231 (2000) 